\title{
The CD44-initiated pathway of T-cell extravasation uses VLA-4 but not LFA-1 for firm adhesion
}

\author{
Mark H. Siegelman, Diana Stanescu, and Pila Estess \\ Laboratory of Molecular Pathology, Department of Pathology, The University of Texas Southwestern Medical Center, \\ Dallas, Texas 75235, USA
}

Address correspondence to: Pila Estess, Department of Pathology, The University of Texas Southwestern Medical Center, 5323 Harry Hines Boulevard, Dallas, Texas 75235-9072, USA.

Phone: (214) 648-4111; Fax: (214) 648-4070; E-mail: pila.estess@email.swmed.edu.

Received for publication October 15, 1999, and accepted in revised form January 24, 2000.

Leukocytes extravasate from the blood in response to physiologic or pathologic demands by means of complementary ligand interactions between leukocytes and endothelial cells. The multistep model of leukocyte extravasation involves an initial transient interaction ("rolling" adhesion), followed by secondary (firm) adhesion. We recently showed that binding of CD44 on activated T lymphocytes to endothelial hyaluronan (HA) mediates a primary adhesive interaction under shear stress, permitting extravasation at sites of inflammation. The mechanism for subsequent firm adhesion has not been elucidated. Here we demonstrate that the integrin VLA-4 is used in secondary adhesion after CD44mediated primary adhesion of human and mouse $T$ cells in vitro, and by mouse $T$ cells in an in vivo model. We show that clonal cell lines and polyclonally activated normal $\mathrm{T}$ cells roll under physiologic shear forces on hyaluronate and require VCAM-1, but not ICAM-1, as ligand for subsequent firm adhesion. This firm adhesion is also VLA-4 dependent, as shown by antibody inhibition. Moreover, in vivo short-term homing experiments in a model dependent on CD44 and HA demonstrate that superantigen-activated T cells require VLA-4, but not LFA-1, for entry into an inflamed peritoneal site. Thus, extravasation of activated T cells initiated by CD44 binding to HA depends upon VLA4-mediated firm adhesion, which may explain the frequent association of these adhesion receptors with diverse chronic inflammatory processes.

J. Clin. Invest 105:683-691 (2000).

\section{Introduction}

Coordinated regulation and appropriate site-specific targeting of leukocyte extravasation results from various types of adhesion receptors acting in sequential fashion, allowing directed leukocyte exit from the blood into diverse tissues within the organism $(1,2)$. Thus, the elucidation of molecules mediating the extravasation of leukocytes into targeted sites is central to the understanding of the complex regulation of the host response to inflammation. Several families of adhesion receptors are thought to be essential in this multistep model of extravasation for both nonspecific effector cells and antigen-specific lymphocytes. The initial contact with and primary adhesion ("rolling") of leukocytes on vascular endothelium has generally been attributed to the engagement of members of the selectin family by their carbohydrate ligands. Subsequent secondary (firm) adhesion is largely due to heterodimeric integrins interacting with their endothelial cell ligands, members of the immunoglobulin gene superfamily $(3,4)$. After arrest, leukocytes migrate through the endothelial and basement membrane barriers into underlying tissues.

Tissue-specific migration pathways of effector and memory T-lymphocyte subsets have been shown to be distinct, and in part attributable to adhesion receptors that they express (5-9). Our laboratory has been engaged in the description and characterization of a pathway of extravasation initiated by CD44 on lymphocytes activated to bind its major ligand, hyaluronan (HA). CD44 interactions with HA expressed on endothelial cells permit lymphocyte rolling under shear conditions simulating physiologic flow (10). Although the mechanism by which CD44 becomes activated to bind HA has not yet been completely elucidated, it has become clear that stimulation of normal $\mathrm{T}$ lymphocytes in vitro or in vivo through the $\mathrm{T}$-cell receptor induces the activated form of CD44 and attendant primary adhesion on endothelium or HA substrate (11-13). These observations have established the HA-binding form of CD44 as an early activation marker on T cells after T-cell receptor stimulation, and support a role for this interaction during the course of an immune response. Moreover, CD44/HA interactions have been shown to be required for extravasation of superantigenstimulated $\mathrm{T}$ cells into an inflamed peritoneal site in a mouse model (12). CD44 has been prominently associated with human arthritis and with a model of collageninduced murine arthritis (14-17), and more recently with a model of multiple sclerosis, experimental allergic encephalomyelitis (18). CD44 interactions with HA are also thought to be important in allogeneic graft rejection $(19,20)$. We have proposed that this CD44-initiated pathway is used primarily by activated $\mathrm{T}$ cells for 
egress into a variety of inflamed tissues. Consistent with this suggestion, the presence of circulating $\mathrm{T}$ cells bearing the activated form of CD44 that enables HAdependent rolling correlates highly with exacerbation of major autoimmune disease in humans (15). Evidence suggests that HA binding through CD44 can be regulated through mechanisms such as glycosylation and a variety of other posttranslational modifications (21-25), but the particular molecular basis for CD44 activation of this subset of T cells has not been clarified.

The predominant interactions observed in laminar flow assays between activated T cells bearing CD44 and an HA substrate have been those of primary adhesion. For physiologic relevance, these interactions would require subsequent firm adhesion and arrest to achieve extravasation, as predicted by our model and by in vivo evidence (12). Because secondary adhesion preceded by selectin-mediated primary adhesion is generally mediated by integrins, we chose to examine the 2 integrins that are most prominently expressed on most circulating T cells - VLA-4 ( $\alpha 4 \beta 1)$ and LFA-1 ( $\alpha$ L $\beta 2)-$ and their ligands as candidates for secondary adhesion subsequent to CD44-mediated rolling. We report that in these studies, VLA-4 is the major integrin used in this pathway both in vitro and in an in vivo model, whereas LFA-1 contributes little to this process.

\section{Methods}

Reagents. PMA and staphylococcal enterotoxin B (SEB) was purchased from Sigma Chemical Co. (St. Louis, Missouri, USA). Rooster comb sodium hyaluronate was from Fisher Scientific Co. (Pittsburgh, Pennsylvania, USA). Ionomycin was from Calbiochem-Novabiochem Corp. (La Jolla, California, USA), and 5,6-carboxyfluorescein diacetate succinimidyl ester (CFDA) came from Molecular Probes Inc. (Eugene, Oregon, USA). Murine TNF- $\alpha\left(5 \times 10^{7} \mathrm{U} / \mathrm{mg}\right)$ was obtained from Genzyme Corp. (Cambridge, Massachusetts, USA).

Cell culture. The murine T-cell line SAKRTLS12 was a gift from P. Kincade (Oklahoma Medical Research Foundation, Oklahoma City, Oklahoma, USA) and J. Lesley (The Salk Institute, La Jolla, California, USA). The human EBV-transformed B-lymphoblastoid cell line KCA (26) was kindly provided by L. Picker of the University of Texas Southwestern Medical Center (UTSWMC). Cells were maintained in DMEM and RPMI-1640, respectively, with high glucose, 10\% FCS, $1 \mathrm{mM}$ pyruvate, and $2 \mathrm{mM}$ glutamine. Human T cells from peripheral blood (HuPBT) of healthy volunteers were obtained by passing mononuclear cells enriched by Ficoll-Hypaque gradient centrifugation over T-cell enrichment columns, according to the manufacturer's instructions (R\&D Systems Inc., Minneapolis, Minnesota, USA). Where indicated, cell lines and HuPBT were activated with $1 \mathrm{ng} / \mathrm{mL}$ PMA plus $500 \mathrm{ng} / \mathrm{mL}$ ionomycin for $18-24$ hours at $37^{\circ} \mathrm{C}$.

Lymph node cells were obtained from BALB/c mice approximately 8 weeks old. Five milliliters of freshly isolated lymph node cells $\left(2 \times 10^{6}\right.$ cells per $\left.\mathrm{mL}\right)$ were incubated at $37^{\circ} \mathrm{C}$ with immobilized hamster antiCD3 (PharMingen, San Diego, California, USA) at 5 $\mu \mathrm{g} / \mathrm{mL}$ for 48 hours.

SVEC4-10 is an SV40-transformed murine lymph node endothelial cell line derived from lymph node stroma. Endothelial cells were maintained in a highglucose solution of RPMI-1640, 15\% FCS, $1 \mathrm{mM}$ pyruvate, $2 \mathrm{mM}$ glutamine, and $50 \mu \mathrm{m} \beta$-mercaptoethanol. For rolling assays, cells were plated at $30 \%$ confluence and then incubated for 48-72 hours on $30-\mathrm{mm}$ tissue culture dishes. TNF- $\alpha(10 \mathrm{ng} / \mathrm{mL})$ was included in the final 18 hours of incubation to optimize levels of surface HA expression (27).

Antibodies and soluble proteins. Hybridomas producing HA-blocking rat anti-mouse CD44 (clone KM81) and blocking rat anti-mouse LFA-1 (clone M17/4) were obtained from the American Type Culture Collection (Manassas, Virginia, USA). Blocking rat anti-mouse ICAM-1 (clone YN1/1.7) was provided by P. Thorpe (UTSWMC). Antibodies were purified from tissue culture supernatants by protein A-Sepharose column chromatography. Rat anti-mouse VLA-4 (anti- $\alpha 4$; clone PS/2) was kindly supplied by P. Kincade. Phycoerythrin (PE) and biotin conjugates of rat anti-mouse CD44 (non-HA blocking clone IM7) were purchased from PharMingen for FACS ${ }^{\circledR}$ analysis.

HA-blocking mouse anti-human CD44 (clone 515) was kindly provided by G. Kansas (Northwestern University Medical School, Chicago, Illinois, USA) $(28,29)$. Blocking mouse anti-human VLA-4 $\alpha$ chain (clone L25) (30) was purified from mouse ascites. Blocking mouse antihuman LFA-1 (clone G43-25B) was purchased from PharMingen. The hybridoma producing blocking mouse anti-human ICAM-1 (clone R6.5.D6.E9.B2) was obtained from the American Type Culture Collection; antibody was purified from culture supernatants as above.

Streptavidin-RED670 (SA-R670) was purchased from GIBCO BRL (Gaithersburg, Maryland, USA). PEconjugated goat anti-rat immunoglobulin (GaRIg-PE) and goat anti-mouse immunoglobulin (GaMIg-PE) were obtained from Caltag Laboratories Inc. (Burlingame, California, USA).

Recombinant soluble human VCAM-1 and ICAM-1 (sVCAM-1 and sICAM-1) were purchased from R\&D Systems Inc. A cell line producing recombinant murine sICAM-1 was a gift from F. Takei (University of British Columbia, Vancouver, Canada). Mouse sICAM-1 was purified at $4^{\circ} \mathrm{C}$ by passing culture supernatant through an anti-ICAM-1 antibody affinity column. The column was extensively washed with $0.15 \mathrm{M} \mathrm{NaCl}$ and $10 \mathrm{mM}$ Tris at $\mathrm{pH}$ 7.5; bound sICAM-1 was eluted with $0.15 \mathrm{M} \mathrm{NaCl}$ and $50 \mathrm{mM}$ diethylamine at $\mathrm{pH}$ 11.0. The eluate was immediately neutralized with a $0.1 \times$ volume of $1 \mathrm{M}$ Tris at $\mathrm{pH}$ 6.0. Eluted material was concentrated and exchanged into PBS using Amicon Centricon 30 microconcentrators (Millipore Corp., Beverly, Massachusetts, USA). The purity of sICAM-1 was confirmed by SDS-PAGE, and the concentration was determined using a DC Protein Assay Kit (Bio-Rad Labora- 
a

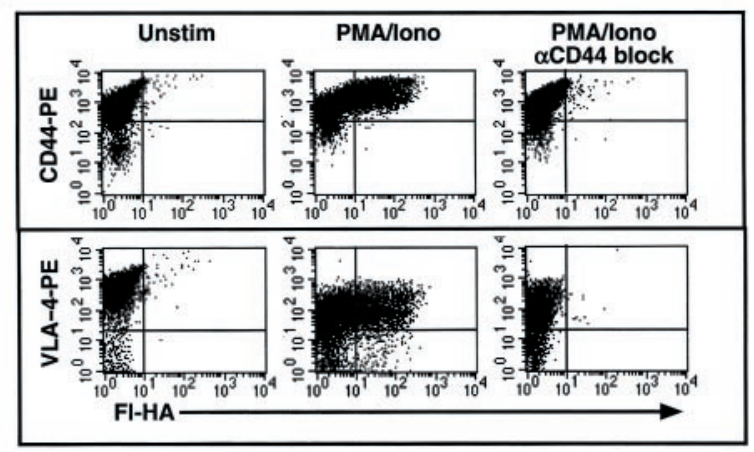

b
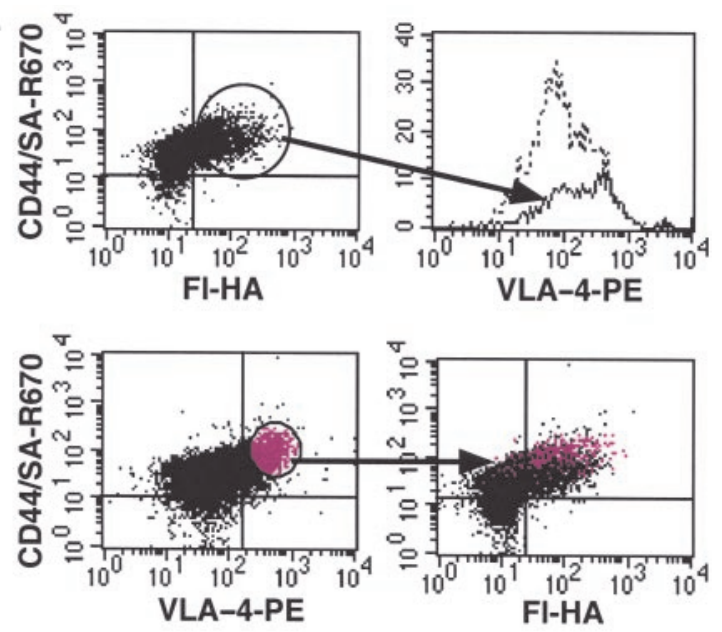

\section{Figure 1}

FI-HA binding by HuPBT. (a) Untreated cells (Unstim) or cells treated with PMA and ionomycin for 18 hours were stained with Fl-HA plus either CD44-PE or VLA-4/GaRIg-PE as indicated, and then analyzed by 2-color FACS ${ }^{\circledR}$. Few Fl-HA ${ }^{+}$cells are seen among untreated HuPBT cells, whereas a distinct population of positive cells is seen after activation. Fl-HA-binding cells are found predominantly in both the CD44 (top) and VLA-4+ (bottom) populations. Fl-HA binding by both CD $44^{+}$and $\mathrm{VLA}-4^{+}$activated $\mathrm{T}$ cells is blocked by incubation with blocking anti-CD44 before staining. (b) Three-color immunofluorescence analysis of activated T cells demonstrates that the CD44 hi/FI-HA ${ }^{+}$population is also the VLA-4hi population. Top: Histograms show the VLA-4-PE staining of cells stained with FI-HA, VLA-4-PE, and CD44/SA-R670. The VLA-4-PE staining of gated CD44/FI-HA double-positive cells (circled) is indicated by the solid histogram; the dashed histogram reflects the VLA-4-PE staining of the total population. Bottom: The CD44/VLA-4 double-positive population (red, circled) is contained predominantly in the CD44/Fl-HA double-positive population.

tories Inc., Hercules, California, USA). Integrity of the purified sICAM-1 was verified by inhibition of anti-ICAM- 1 staining of ICAM- $1^{+}$cells and by LFA1 -dependent adhesion of activated murine $T$ cells on sICAM-1-coated dishes in a static adhesion assay as described $(10,27)$ (data not shown).

FACS ${ }^{\circledR}$ analysis. Unstimulated or PMA and ionomycin-activated lymphoid cells $\left(5 \times 10^{5}\right)$ were stained with antibody for 10 minutes on ice and then washed with $1 \mathrm{~mL}$ of PBS and 5\% FCS. When indicated, cells were incubated with secondary antibody or SA-R670 for an additional 10 minutes and washed again. For blocking studies, a saturating amount of unlabeled monoclonal antibody 515 (which does not cross-block the staining of IM7) was preincubated with cells before addition of fluoresceinated-HA (Fl-HA). Data were collected on a FACScan (Becton Dickinson Immunocytometry Systems, San Jose, California, USA) and were analyzed using CellQUEST 3.1 software (Becton Dickinson Immunocytometry Systems).

Adhesion assay under flow conditions. Rolling and firm adhesion of lymphocytes was assessed on a parallel plate flow chamber under physiologic flow conditions as described (10). Briefly, a 35-mm tissue culture dish (Corning Glass Works, Corning, New York, USA) was coated with $1 \mathrm{~mL}$ of $2.5 \mathrm{mg} / \mathrm{mL}$ HA in PBS overnight at $4^{\circ} \mathrm{C}$ or with $1 \mathrm{~mL}$ sVCAM- $1(0.5-1.0 \mu \mathrm{g} / \mathrm{mL})$ or sICAM-1 $(1 \mu \mathrm{g} / \mathrm{mL})$ in a solution of $10 \mathrm{mM} \mathrm{NaHCO}$ in PBS ( $\mathrm{pH}$ 9.1) for 2 hours at room temperature on a rocking platform. Plates coated with HA plus sVCAM1 or HA plus sICAM-1 were first incubated overnight with HA followed by a 2-hour incubation with sVCAM-1 or sICAM-1. Plates were blocked with $1 \mathrm{~mL}$ FCS for 1 hour at $4^{\circ} \mathrm{C}$ before use, and then rinsed 3 times with PBS. No tethering, rolling, or firm interactions were seen on plates coated with VCAM-1 or ICAM-1 alone at these concentrations, consistent with previous reports perfusing $\mathrm{T}$ cells at physiologic shear forces (1.8 dynes $/ \mathrm{cm}^{2}$ ) over VCAM-1-coated plates at concentrations up to $15 \mu \mathrm{g} / \mathrm{mL}$ (31). Direct firm adhesion in this system was only detectable on plates coated with sVCAM-1 alone at concentrations above 3 $\mu \mathrm{g} / \mathrm{mL}$. Monolayers of SVEC4-10 endothelial cells were used at confluence. Experiments were carried out at a wall shear stress of 2.0 dynes $/ \mathrm{cm}^{2}$ for mouse cells or 1.7 dynes $/ \mathrm{cm}^{2}$ for human cells. Blocking studies were carried out by incubating the cells with saturating concentrations of antibody for 10 minutes before the rolling assay.

For quantitation, rolling cells from at least 4 separate fields were counted for 1-2 minutes per field. Firm adhesion (sticking) was determined by counting total firmly adherent cells on 4 fields after 4 minutes of flow. At least 3 samples were analyzed for each interaction condition. The average was calculated and results are shown as percentage of positive control interaction (rolling or sticking). For experiments on endothelial cell monolayers, the data are expressed as cells/field per minute (rolling) and bound cells/field (sticking).

Short-term homing of lymphocytes in vivo. BALB/c mice were raised in the animal colony at UTSWMC. All in vivo experimental groups consisted of 3 or more mice. Donor 
a

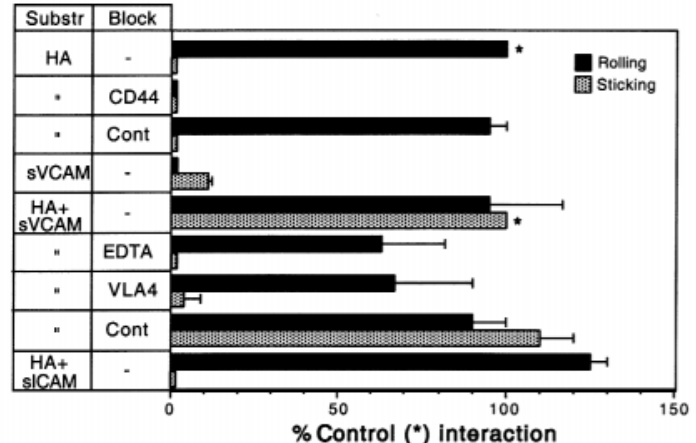

C

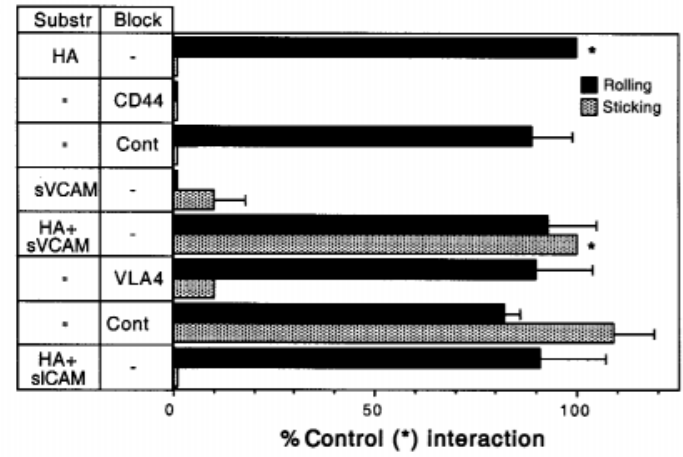

e

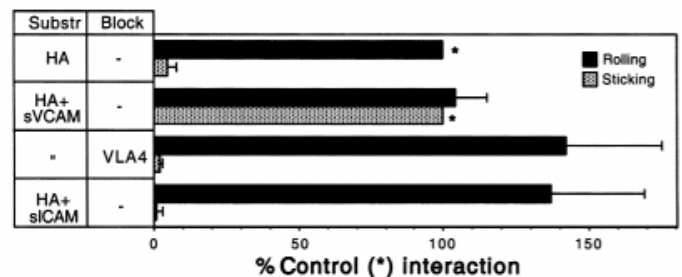

b

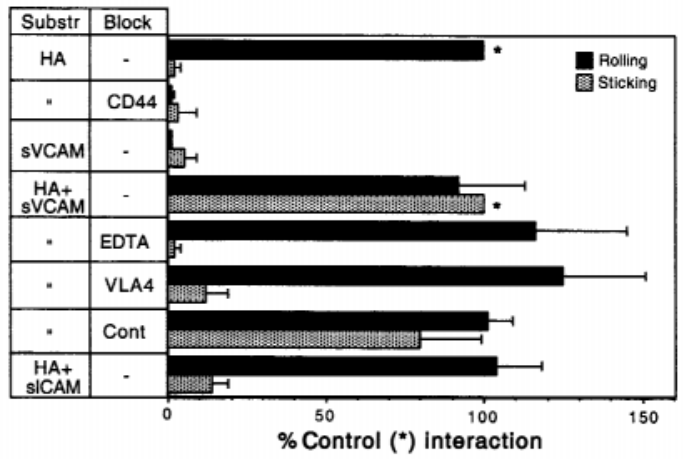

d

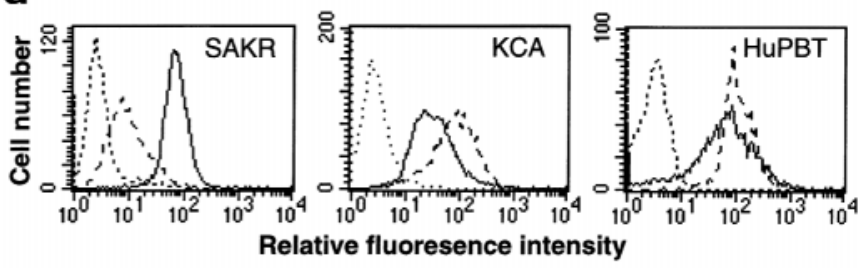

Figure 2

Primary (rolling) and secondary (firm) adhesion of murine SAKR (a), human KCA (b), and HuPBT (c) cells on soluble substrates under physiologic shear stress. Cells were applied to feed solution already equilibrated under flow and then perfused over the indicated individual or combined substrates in the parallel plate flow assay. Rolling and sticking were analyzed as described in Methods, and the number of rolling cells/field per minute and the number of bound cells/field were determined for each substrate. Results are compared to control interactions (indicated with asterisks), and presented as a percentage of the total (100\%) control. Mean absolute values (100\%) for rolling (given in cells/field per minute) and sticking (given in cells/field), respectively, were: SAKR, 14.6 and 9.7; KCA, 40 and 35.5; HuPBT, 9.6 and 9.8. For blocking, antiCD44, anti-VLA-4, anti-LFA-1 (Cont), or EDTA was added to the cell suspension before introduction into the flow system. With each cell type, firm adhesion occurred only on plates coated with HA plus sVCAM-1. All interactions were blocked with anti-CD44, whereas only anti-VLA-4 was able to block sticking without affecting rolling. EDTA also inhibited firm adhesion. (d) Expression of integrins VLA-4 and LFA-1 on SAKR, KCA, and HuPBT cells. Cells were stained with primary antibody plus GaRIg-PE or GaMlg-PE for FACS ${ }^{\circledR}$ analysis. All 3 cell types were positive for both VLA-4 (dashed lines) and LFA-1 (solid lines). Stippled lines represent control staining. (e) Primary and secondary adhesion of antiCD3-activated murine lymph node cells on soluble substrates under physiologic shear stress. Assays were performed and analyzed as above. Mean absolute values (100\%) for rolling and sticking, respectively, were 15.1 cells/field per minute and 72.8 cells/field. Substr, substrate; Block, blocking antibody or EDTA.

and recipient mice, $8-12$ weeks old, were injected with 50 $\mu \mathrm{g}$ SEB in $500 \mu \mathrm{L}$ PBS or with PBS alone. Lymphocytes from mesenteric lymph nodes of donor mice were isolated, labeled with CFDA for 20 minutes at room temperature, and washed with PBS. Twenty hours after SEB injection of recipient mice, $10^{7}$ fluorescent donor cells in $500 \mu \mathrm{L}$ HBSS were injected into the tail vein of each recipient mouse. For blocking experiments, antibody was added at saturating concentration $(100 \mu \mathrm{g})$ to the cell suspension before injection. Ninety minutes after intravenous cell injection, recipient mice were sacrificed. All cells in the peritoneal cavity were collected by lavage with $5 \mathrm{~mL}$ of RPMI- 1640 at $37^{\circ} \mathrm{C}$, and were analyzed by FACS ${ }^{\circledR}$ for the presence of fluorescent donor cells. At least 10 animals were analyzed for each condition.

\section{Results}

The activated form of CD44 is associated with elevated VLA-4 expression on activated leukocytes. The 3 cell types used for these studies - the murine T cell line SAKRTLS12, the 
human lymphoblastoid line KCA, and normal HuPBT - were chosen based on their coexpression of the common leukocyte integrins $\alpha 4 \beta 1$ (VLA-4) and $\alpha \mathrm{L} \beta 2$ (LFA1 ), as well as for their ability to convert CD44 to its activated (HA-binding) form after polyclonal stimulation $(10,32)$. Although CD44 is expressed at significant levels on resting HuPBT, it does not bind Fl-HA (Figure 1a). After overnight polyclonal stimulation, a significant percentage of the $\mathrm{CD} 44^{+}$cell population acquires the ability to bind Fl-HA $(10,32)$. As shown, the number of cells with the Fl-HA-binding phenotype was particularly increased among the VLA- $4^{+}$cells after stimulation, although overall VLA-4 levels diminished somewhat (Figure 1a). Thus, activation of CD44 to the HA-binding form is preferentially associated with cells expressing higher levels of VLA-4. This is further detailed in Figure 1b, where 3-color analysis demonstrates that CD $44^{+}$Fl-HA-binding cells express VLA-4 at higher levels than are seen in the total population (top). Likewise, it can be seen that CD $44^{\text {hi }} / \mathrm{VLA}-4^{\text {hi }}$ double-positive cells are predominantly positive for Fl-HA binding (bottom), a finding also seen in SEB-stimulated mouse lymphocytes (see Figure 4a).

CD44/HA-mediated primary adhesion is followed by VLA4/VCAM-1-dependent secondary adhesion under shear forces. To examine firm adhesion directly under laminar flow after CD44-initiated rolling adhesion, plates were coated with HA simultaneously with the VLA-4 and LFA-1 integrin ligands sVCAM-1 and sICAM-1, respectively. Thus, activated murine and human lines and normal human $\mathrm{T}$ cells were analyzed for both primary and secondary adhesion under laminar flow on the substrates HA, sVCAM-1, HA plus sVCAM-1, and HA plus sICAM1. Results of these analyses are shown in Figure 2. Activated SAKR cells exhibit primary adhesion on plates coated with $\mathrm{HA}$ alone, and this rolling can be completely inhibited with blocking anti-CD44 antibody, but not with isotype-matched control anti-LFA-1 (Figure 2a) (10). Both of the human cell populations (KCA and HuPBT) also rolled on HA after stimulation with PMA and ionomycin; this was also CD44 dependent (Figure $2, \mathrm{~b}$ and $\mathrm{c}$ ). No substantial firm adhesion was noted with any of the cells tested on HA substrate alone, again confirming that the CD44/HA interaction is involved primarily in rolling adhesions.

On sVCAM-1 alone, detectable but minimal direct firm adhesion, and essentially no rolling, was observed (Figure 2). In contrast, when each of these cell populations was assayed on a substrate of HA plus SVCAM-1, significant numbers of firmly adherent cells were seen in addition to rolling cells (Figure 2, a-c), indicating that sVCAM-1 can function as a secondary adhesion ligand after primary adhesion mediated by CD44/HA. In addition, $22-46 \%$ of total adherent cells at 4 minutes were observed to convert from rolling to firmly adherent. To confirm that the lymphocyte receptor for this interaction was the integrin VLA-4, cells were pretreated with anti-VLA-4 before the shear stress assay. Anti-VLA-4 antibody treatment (M17/4 for mouse cells and L25 for human cells) completely inhibited firm adhesion in each case, but had little effect on CD44-dependent rolling. Control antibody also had no effect on either rolling or sticking. Also consistent with an integrin-mediated interaction (which requires the presence of divalent cations) is the fact that firm adhesion was preferentially inhibited by inclusion of $5 \mathrm{mM}$ EDTA in the assay buffer (Figure 2, a and b). Moreover, the VLA-4 dependence of firm adhesion was not due to differences between expression levels of VLA-4 and LFA-1, because each cell type expressed comparable levels of both integrins, with SAKR cells expressing significantly more LFA-1 than VLA-4 (Figure 2d). In contrast, no firm adhesion was seen on plates coated with both HA and sICAM-1. Neither direct firm adhesion of cells to sICAM-1 alone nor firm adhesion after CD44mediated primary adhesion on plates coated with both HA and sICAM-1 was observed, even at 10-fold higher sICAM-1 concentrations (data not shown). Thus, although expressed on all 3 cell types, LFA- 1 does not appear to play a significant role in mediating firm adhesion under shear stress in the CD44-initiated pathway. However, it should be noted that LFA-1 on these populations was competent to bind ICAM-1 on coated plates in both static and nonstatic (rotational, low shear) adhesion assays $(27,33)$ (data not shown), consistent with prior reports (34-38). It is also of note that for these activated lymphocyte populations,

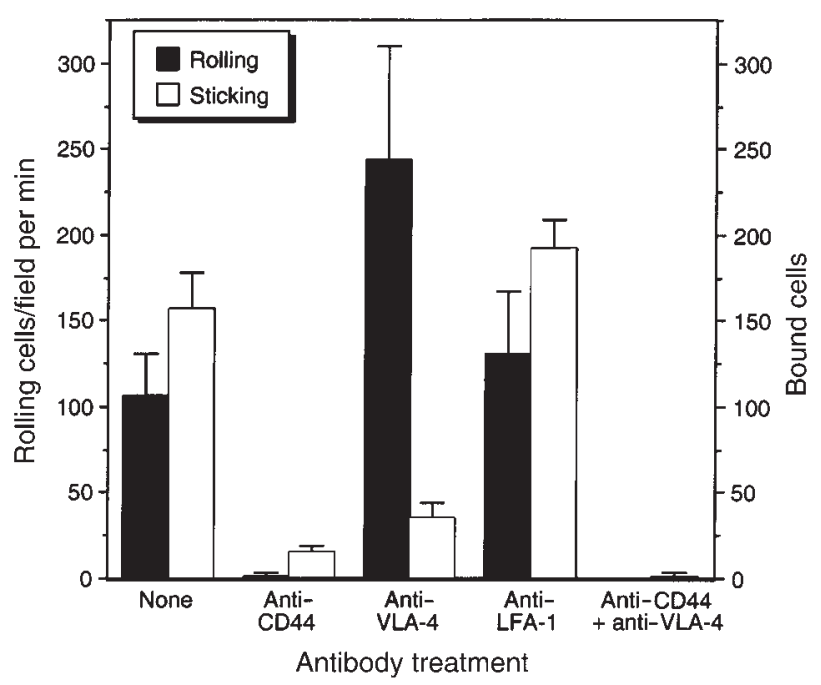

\section{Figure 3}

Primary and secondary adhesion of SAKR cells on SVEC4-10 endothelial cells under physiologic shear stress. SAKR cells were applied to feed solution already equilibrated under flow at a wall shear stress of 2.0 dynes $/ \mathrm{cm}^{2}$ and the solution was perfused over the monolayer in the parallel plate flow assay. Rolling and sticking were analyzed as described, and the number of cells/field per minute rolling and number of bound cells/field were determined. For blocking, anti-CD44, anti-VLA-4, or anti-CD44 together with anti-VLA-4 was added to the cell suspension before introduction into the flow system. Rolling and firm adhesion were both blocked by anti-CD44, whereas only anti-VLA-4 was able to block firm adhesion. Data shown are from a representative of 3 experiments. 
a

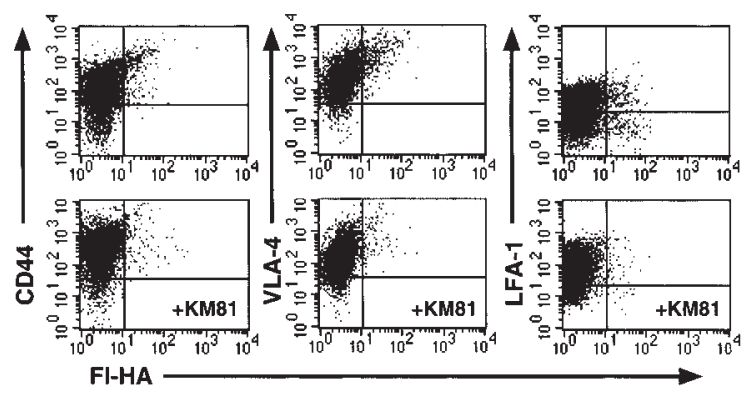

b

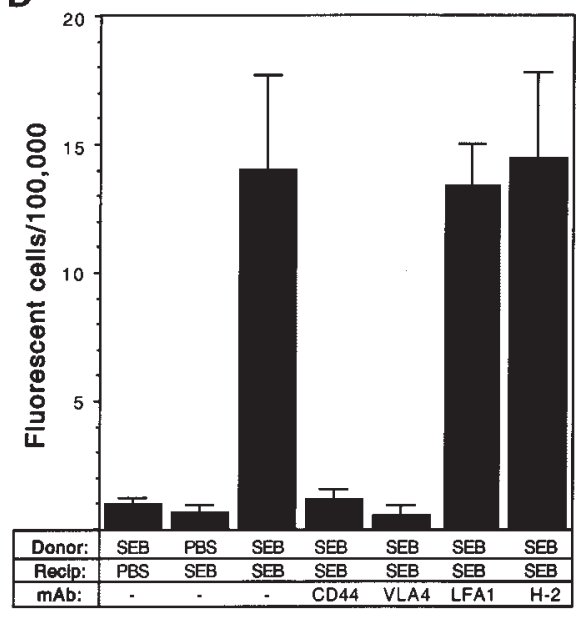

Figure 4

VLA-4 participates in the short-term homing of activated lymphocytes to an inflamed site. (a) Mesenteric lymph node cells from SEB-treated mice were stained with Fl-HA plus either CD44-PE, VLA-4/GaRIg-PE, or LFA-1/GaRIg-PE as indicated. Fl-HA-binding cells are present predominantly in both the CD $44^{+}$and $\mathrm{VLA}-4^{+}$populations, but are distributed between both $\mathrm{LFA}-1^{+}$and $\mathrm{LFA}-1^{-}$populations. Fl-HA binding is blocked by incubation with blocking anti-CD44 before staining. (b) CFDA-labeled mesenteric lymph node cells from SEB- or PBS-treated mice were injected intravenously into recipient mice that had received an intraperitoneal injection of SEB 20 hours earlier. Cells from donor mice were injected without antibody or with HA-blocking anti-CD44 monoclonal antibody, blocking anti-VLA-4 $\alpha$, blocking anti-LFA1, or isotype-matched control anti-H-2 monoclonal antibody. Two hours after donor cell injection, peritoneal cavities of recipient mice were lavaged, and recovered cells were analyzed by FACS ${ }^{\circledR}$ for CFDA fluorescence. Data are shown as the number of fluorescent cells per 100,000 cells analyzed, and represent the mean \pm SEM of 3 separate experiments ( $\geq 3$ mice/group per experiment). No peritoneal accumulation of fluorescent cells was seen when either anti-CD44 or anti-VLA-4 antibody was added to the injected cell suspension. Anti-LFA-1 and control anti-H-2 had no effect on cell migration into peritoneal cavities.

rolling mediated by VLA-4/VCAM-1 was only minimally observed, although this capability has been demonstrated in previous studies $(31,39,40)$. However, our observations are consistent with prior reports that rolling was not observed on isolated VCAM-1 substrate using activated lymphoid cells. Immediate firm adhesion was seen, but only at lower shear forces (0.3-0.7 dynes $\left./ \mathrm{cm}^{2}\right)$ (31). Direct firm adhesion was not observed on VCAM- 1 under the conditions used in these studies. Therefore, using soluble substrates, primary adhesion mediated by activated CD44/HA appears to be followed by secondary adhesion mediated by VLA-4/VCAM-1 under physiologic shear stresses; this sequence is maintained in both mouse and human.

To determine whether a more physiologic stimulus resulted in a similar adhesion pathway, murine lymph node cells were stimulated directly through the T-cell receptor with anti-CD3. This alternative stimulation resulted in a pattern that was virtually identical to that seen with PMA and ionomycin. Stable arrest was overwhelmingly dependent on VLA-4 after CD44-initiated rolling adhesion (Figure 2e). Thus, a more physiologic protocol confirms the pattern found with the above populations.

VLA-4/VCAM-1-mediated firm adhesion follows CD44/ $H A$ primary adhesion on endothelial monolayers. We have previously demonstrated CD44-mediated rolling of Tcell populations on endothelial cell lines and primary endothelial cultures expressing HA. Moreover, endothelial HA levels are induced by proinflammatory stimuli; this results in increased CD44/HA-mediated adhesive interactions under conditions simulating physiologic shear stress $(10,27,41)$. However, only primary adhesion was addressed in those experiments. Therefore, we sought to confirm the observations on isolated substrate, and to further examine the role of VLA-4-mediated secondary adhesion after CD44-mediated primary adhesion on endothelial monolayers expressing VCAM-1. To this end, we tested activated SAKR cells under laminar flow on monolayers of TNF$\alpha$-treated murine SVEC4-10 endothelial cells, which express both VCAM-1 and ICAM-1 (41). As shown in Figure 3, both primary and firm adhesion of SAKR cells occurred on this monolayer. Essentially all rolling interactions were blocked by anti-CD44, and very little firm adhesion was evident when CD44-mediated rolling was blocked, indicating a minimal role for VLA4 in primary adhesion on these endothelial cells. Furthermore, very little firm adhesion was observed when CD44 interactions were blocked, indicating a minimal role of VLA-4/VCAM-1 in direct firm adhesion in this system. In contrast, firm adhesion was blocked more than $80 \%$ by anti-VLA- 4 , with compensatory increases in rolling interactions, whereas the control anti-LFA-1 (also expressed on SAKR cells, Figure 2d) had no significant effect on either sticking or rolling. A combination of anti-CD44 and anti-VLA-4 removed almost all interactions. Thus, the adhesion interactions observed 
using soluble substrates can be reproduced under more physiologic circumstances using endothelial cells expressing the appropriate ligands, HA and VCAM-1.

VLA-4 participates in boming of superantigen-activated lymphocytes to an inflamed site in vivo. We showed previously that activated CD44 interactions with HA are critical to the traffic of superantigen-activated $\mathrm{T}$ cells from the bloodstream into inflamed peritoneal cavities in a shortterm homing assay $(13,41)$. We conducted similar studies to determine if VLA-4 on lymphocytes also participates in this in vivo trafficking model. Draining mesenteric lymph node cells, from mice treated with SEB 20-24 hours before sacrifice, were used as donor cells. FACS $^{\circledR}$ analysis of these cells demonstrated a subpopulation bearing activated CD44, and a similar population expressing high levels of VLA-4 that preferentially bound Fl-HA (Figure 4a). Furthermore, as with activated normal human T cells (Figure 1), the HA-binding cells were concentrated in the population coexpressing high levels of both CD44 and VLA-4 (data not shown). In contrast, HAbinding cells were more evenly distributed across the expression levels of LFA-1, similar to results seen with activated human $T$ cells (data not shown).

To assess homing capabilities, CFDA-labeled activated donor lymph node cells were injected intravenously into recipient mice treated intraperitoneally with SEB to create an inflamed peritoneal site. Migration of fluorescent donor cells from the blood into the inflamed site was assessed by FACS $^{\circledR}$ analysis of cells recovered by lavage of the peritoneal cavity 2 hours after intravenous injection. As shown in Figure 4b, significant numbers of fluorescent donor cells were found in recipient peritoneal cavities compared with either unactivated donor cells or activated cells injected into untreated recipients; these cells have previously been shown to be the activated V $\beta 8$-bearing T-cell subset $(12,41)$. Fluorescent donor-cell migration into recipient peritoneal cavities is almost completely prevented by coadministration of either blocking anti-CD44 antibody or blocking anti-VLA-4 antibody (Figure 4b). However, neither anti-LFA-1 nor control anti-H-2 antibodies had any effect on cell migration. Thus, in an in vivo homing model that depends on CD44/HA interactions, VLA-4 also appears to be essential in the trafficking of activated lymphocytes into an inflamed peritoneal site, consistent with a model in which CD-44 and VLA-4 act sequentially.

\section{Discussion}

VLA-4 is expressed on T cells, monocytes, and eosinophils (and according to some studies, on neutrophils), and has been shown to have a role in many inflammatory models involving all of these cell types (42-48). The VLA-4/VCAM-1 interaction has been shown to be important for lymphocyte/endothelial interactions at several inflammatory sites. Examples include entry of $\mathrm{CD} 4^{+} \mathrm{T}$ cells into the brain in experimental allergic encephalomyelitis $(42,49)$, in rheumatoid synovium $(50,51)$, and for the development of autoimmune diabetes (52). Our prior evidence in an antigen-specific contact hypersensitivity response suggested that this integrin operates at the level of effector $\mathrm{T}$ cells, which are in turn necessary for the recruitment of nonspecific effector cells (53). This is consistent with other contact hypersensitivity studies in mice and primates (54-56), while in a rat model of skin delayed-type hypersensitivity, both anti-VLA- 4 and LFA-1 were required for maximal in vivo blocking (57). The studies presented here suggest that the lymphocyte-trafficking roles attributed to VLA-4 could in some situations follow CD44 tethering and rolling on endothelial HA.

Integrins containing the $\alpha 4$ chain have been demonstrated to be quite versatile with respect to the adhesion cascade. In addition to firm arrest (4), these integrins are able to mediate tethering and rolling. In contrast, we observed no measurable rolling, although, as noted, we did detect some direct attachment on VCAM-1 alone (Figure 2), consistent with earlier observations (31). In addition, $\alpha 4 \beta 7$ could mediate firm arrest but not rolling of preactivated lymph node cells in Peyer's patch high endothelial venules in vivo (58). Analogous to the CD44-initiated pathway described here, VLA-4 has been reported to mediate firm adhesion of $\mathrm{CD}^{+} \mathrm{T}$ cells after P-selectin-driven primary adhesion (59). Moreover, in some in vivo models, rolling mediated by $\alpha 4$ and integrins/VCAM- 1 has been shown to have a detectable but very small role compared with all selectin interactions (60). Thus, the CD44/HA primary adhesion interaction may be preferentially used in conjunction with VLA-4 by activated cell populations, whereas resting cells on which CD44 is not activated may use $\alpha 4$ integrins for both adhesion steps during extravasation, or may act in conjunction with selectins to effect firm adhesion.

The predilection for the use of VLA- 4 but not LFA- 1 in these experiments is striking. Very little contribution by LFA-1 under laminar flow (Figures 2 and 3 ) or for in vivo homing (Figure 4) could be detected, even though it was clear that LFA-1 was active after cell stimulation in static binding assays to purified mouse or human SICAM-1 (data not shown). This latter point is in agreement with previous reports showing active LFA- 1 after cell stimulation (34-36, 61, 62). In addition, in laminar flow analysis applying half the shear used in other experiments, direct firm adhesion could be detected on ICAM-1 alone (data not shown). This further suggests that LFA-1 on recently activated cells could potentially participate in secondary adhesion after primary adhesion is initiated. LFA- 1 is essential for the homing of naive lymphocytes into peripheral lymph nodes after L-selectin-mediated tethering and rolling (63). Thus, LFA-1 may generally be used for recruitment of naive rather than activated $T$ cells. The mechanism for this selectivity requires further investigation; however, cross-talk between integrins has been reported. For example, interaction of LFA-1 selectively reduces adhesion mediated by $\alpha 4 \beta 1$, allowing coordination of integrin function on $\mathrm{T}$ cells (37), although our evidence suggests the reverse: that $\alpha 4 \beta 1$ may suppress LFA- 1 in recently activated lymphoid cells. 
In summary, these studies were undertaken to examine the mechanism by which CD44/HA-mediated tethering and rolling converts to firm adhesion. Both mouse and human activated $\mathrm{T}$-cell populations demonstrate a dominant role for VLA-4 in this step under conditions of laminar flow, and superantigen-activated $\mathrm{T}$ cells show dependence on both VLA-4 and CD44 in an in vivo mouse model of peritoneal inflammation. LFA-1 does not appear to participate substantially in this pathway in these systems. These results help to clarify the molecular basis for extravasation of activated T cells, which are critical to the initiation and perpetuation of autoimmune and chronic inflammatory states. They may further explain the frequent association of both CD44 and VLA-4 interactions with chronic inflammatory states and suggest that therapeutic strategies targeting both of these receptors may be beneficial.

\section{Acknowledgments}

We thank Heather DeGrendele, and Todd Gonitzke for technical assistance. This work was supported by grants from the National Institutes of Health (R01 CA-57571 and HL-56746) and the Arthritis Foundation. M.H. Siegelman is an Established Investigator of the American Heart Association and a recipient of a Clinical Scientist Award from the Burroughs Wellcome Fund.

1. Butcher, E.C., and Picker, L.J. 1996. Lymphocyte homing and homeostasis. Science. 272:60-66.

2. Springer, T.A. 1994. Traffic signals for lymphocyte recirculation and leukocyte emigration: the multistep paradigm. Cell. 76:301-314.

3. Kansas, G.S. 1996. Selectins and their ligands: current concepts and controversies. Blood. 88:3259-3287.

4. Carlos, T.M., and Harlan, J.M. 1994. Leukocyte-endothelial adhesion molecules. Blood. 84:2068-2101.

5. Mackay, C.R. 1991. T-cell memory: the connection between function, phenotype and migration pathways. Immunol. Today. 12:189-192.

6. Koster, F.T., and McGregor, D.D. 1971. The mediator of cellular immunity. III. Lymphocyte traffic from the blood into the inflamed peritoneal cavity. J. Exp. Med. 133:864-876.

7. Guy, G.D., Griscelli, C., and Vassalli, P. 1978. The mouse gut T lymphocyte, a novel type of $T$ cell. Nature, origin, and traffic in mice in normal and graft-vs.-host conditions. J. Exp. Med. 148:1661-1677.

8. Issekutz, T.B. 1991. Inhibition of in vivo lymphocyte migration to inflammation and homing to lymphoid tissues by the TA-2 monoclonal antibody. A likely role for VLA-4 in vivo. J. Immunol. 147:4178-4184.

9. Austrup, F., et al. 1997. P- and E-selectin mediate recruitment of T-helper1 but not T-helper-2 cells into inflamed tissues. Nature. 385:81-83.

10. DeGrendele, H.C., Estess, P., Picker, L.J., and Siegelman, M.H. 1996. CD44 and its ligand hyaluronate mediate rolling under physiologic flow: a novel lymphocyte/endothelial cell primary adhesion pathway. J. Exp. Med. 183:1119-1130.

11. Lesley, J., Howes, N., Perschl, A., and Hyman, R. 1994. Hyaluronan binding function of CD44 is transiently activated on T cells during an in vivo immune response. J. Exp. Med. 180:383-387.

12. DeGrendele, H.D., Estess, P., and Siegelman, M.H. 1997. Requirement for CD44 in activated T cell extravasation into an inflamed site. Science. 278:672-675.

13. DeGrendele, H.C., Estess, P., and Siegelman, M.H. 1997. CD44 activation and associated primary adhesion is inducible via $\mathrm{T}$ cell receptor stimulation. J. Immunology. 159:2549-2553.

14. Haynes, B.F., Hale, L.P., Patton, K.L., Martin, M.E., and McCallum, R.M. 1991. Measurement of an adhesion molecule as an indicator of inflammatory disease activity. Up-regulation of the receptor for hyaluronate (CD44) in rheumatoid arthritis. Arthritis Rheum. 34:1434-1443.

15. Estess, P., DeGrendele, H.C., Pascual, V., and Siegelman, M.H. 1998 Functional activation of lymphocyte CD44 in peripheral blood is a marker of autoimmune disease activity. J. Clin. Invest. 102:1173-1182.

16. Mikecz, K., Brennan, F.R., Kim, J.H., and Glant, T.T. 1995. Anti-CD44 treatment abrogates tissue oedema and leukocyte infiltration in murine arthritis. Nat. Med. 1:558-563.
17. Verdrengh, M., Holmdahl, R., and Tarkowski, A. 1995. Administration of antibodies to hyaluronanreceptor (CD44) delays the start and ameliorates the severity of collagen II arthritis. Scand. J. Immunol. 42:353-358.

18. Brocke, S., Piercy, C., Steinman, L., Weissman, I.L., and Veromaa, T. 1999. Antibodies to CD44 and integrin alpha(4), but not L-selectin, prevent central nervous system inflammation and experimental encephalomyelitis by blocking secondary leukocyte recruitment. Proc. Natl. Acad. Sci. USA. 96:6896-6901.

19. Knoflach, A., et al. 1999. Immunomodulatory functions of low-molecular weight hyaluronate in an acute rat renal allograft rejection model. J. Am. Soc. Nephrol. 10:1059-1066.

20. Knoflach, A., et al. 1999. Immunomodulatory functions of hyaluronate in the LEW-to-F344 model of chronic cardiac allograft rejection. Transplantation. 67:909-914.

21. Hathcock, K.S., Hirano, H., Murakami, S., and Hodes, R.J. 1993. CD44 expression on activated B cells. Differential capacity for CD44-dependent binding to hyaluronic acid. J. Immunol. 151:6712-6722.

22. Katoh, S., Zheng, Z., Oritani, K., Shimozato, T., and Kincade, P.W. 1995. Glycosylation of CD44 negatively regulates its recognition of hyaluronan. J. Exp. Med. 182:419-429.

23. Lesley, J., English, N., Perschl, A., Gregoroff, J., and Hyman, R. 1995. Variant cell lines selected for alterations in the function of the hyaluronan receptor CD44 show differences in glycosylation. J. Exp. Med. 182:431-437.

24. Bennett, K.L., et al. 1995. Regulation of CD44 binding to hyaluronan by glycosylation of variably spliced exons. J. Cell Biol. 131:1623-1633.

25. Bartolazzi, A., Nocks, A., Aruffo, A., Spring, F., and Stamenkovic, I. 1996. Glycosylation of CD44 is implicated in CD44-mediated cell adhesion to hyaluronan. J. Cell Biol. 132:1199-1208.

26. Goldstein, L.A., et al. 1989. A human lymphocyte homing receptor, the hermes antigen, is related to cartilage proteoglycan core and link proteins. Cell. 56:1063-1072.

27. Mohamadzadeh, M., DeGrendele, H.C., Estess, P., and Siegelman, M.H. 1998. Proinflammatory stimuli regulate endothelial hyaluronan expression and CD44/HA dependent primary adhesion. J. Clin. Invest. 101:97-108.

28. Cannistra, S.A., et al. 1993. Binding of ovarian cancer cells to peritoneal mesothelium in vitro is partly mediated by CD44H. Cancer Res. 53:3830-3838.

29. Kansas, G.S., Wood, G.S., and Dailey, M.O. 1989. A family of cell-surface glycoproteins defined by a putative anti-endothelial cell receptor antibody in man. J. Immunol. 142:3050-3057.

30. Clayberger, C., et al. 1987. Identification and characterization of two novel lymphocyte function-associated antigens, L24 and L25. J. Immunol. 138: $1510-1514$

31. Alon, R., et al. 1995. The integrin VLA-4 supports tethering and rolling in flow on VCAM-1. J. Cell Biol. 128:1243-1253.

32. Lesley, J., Schulte, R., and Hyman, R. 1990. Binding of hyaluronic acid to lymphoid cell lines is inhibited by monoclonal antibodies against PgP1. Exp. Cell Res. 187:224-233

33. Alon, R., Rossiter, H., Wang, X.H., Springer, T.A., and Kupper, T.S. 1994 Distinct cell surface ligands mediate $\mathrm{T}$ lymphocyte attachment and rolling on $\mathrm{P}$ and $\mathrm{E}$ selectin under physiological flow. J. Cell Biol. 127:1485-1495.

34. Schriever, F., et al. 1997. Human T lymphocytes bind to germinal centers of human tonsils via integrin alpha4/VCAM-1 and LFA-1/ICAM-1 and -2. Eur. J. Immunol. 27:35-39.

35. Shimizu, Y., et al. 1991. Four molecular pathways of T cell adhesion to endothelial cells: roles of LFA-1, VCAM-1, and ELAM-1 and changes in pathway hierarchy under different activation conditions. J. Cell Biol. 113:1203-1212.

36. van Kooyk, Y., van de Wiel-van Kemenade, E., Weder, P., Huijbens, R.J., and Figdor, C.G. 1993. Lymphocyte function-associated antigen $1 \mathrm{dom}$ inates very late antigen 4 in binding of activated $T$ cells to endothelium. J. Exp. Med. 177:185-190.

37. Porter, J.C., and Hogg, N. 1997. Integrin cross talk: activation of lymphocyte function-associated antigen- 1 on human T cells alters alpha4beta1and alpha5beta1-mediated function. J. Cell Biol. 138:1437-1447.

38. Jones, D.A., Mcintire, L.V., Smith, C.W., and Picker, L.J. 1994. A two-step adhesion cascade for $T$ cell endothelial cell interactions under flow conditions. J. Clin. Invest. 94:2443-2450.

39. Berlin, C., et al. 1995. Alpha 4 integrins mediate lymphocyte attachment and rolling under physiologic flow. Cell. 80:413-422.

40. Johnston, B., Issekutz, T.B., and Kubes, P. 1996. The alpha 4-integrin supports leukocyte rolling and adhesion in chronically inflamed postcapillary venules in vivo. J. Exp. Med. 183:1995-2006.

41. Estess, P.E., Nandi, A., and Siegelman, M.H. 1999. IL-15 induces endothelial hyaluronan expression in vitro and promotes activated $\mathrm{T}$ cell extravasation through a CD44 dependent pathway in vivo. J. Exp. Med. 190:9-19.

42. Yednock, T.A., et al. 1992. Prevention of experimental autoimmune encephalomyelitis by antibodies against alpha 4 beta 1 integrin. Nature. 356:63-66 
43. Issekutz, A.C., and Issekutz, T.B. 1995. Monocyte migration to arthritis in the rat utilizes both CD11/CD18 and very late activation antigen 4 integrin mechanisms. J. Exp. Med. 181:1197-1203.

44. Issekutz, T.B., and Issekutz, A.C. 1991. T lymphocyte migration to arthritic joints and dermal inflammation in the rat: differing migration patterns and the involvement of VLA-4. Clin. Immunol. Immunopathol. 61:436-447.

45. Yang, X.D., Karin, N., Tisch, R., Steinman, L., and McDevitt, H.O. 1993. Inhibition of insulitis and prevention of diabetes in nonobese diabetic mice by blocking L-selectin and very late antigen 4 adhesion receptors. Proc. Natl. Acad. Sci. USA. 90:10494-10498.

46. Issekutz, T.B., Miyasaka, M., and Issekutz, A.C. 1996. Rat blood neutrophils express very late antigen 4 and it mediates migration to arthritic joint and dermal inflammation. J. Exp. Med. 183:2175-2184.

47. Andersson, E.C., Christensen, J.P., Marker, O., and Thomsen, A.R. 1994. Changes in cell adhesion molecule expression on T cells associated with systemic virus infection. J. Immunol. 152:1237-1245.

48. Reinhardt, P.H., Elliott, J.F., and Kubes, P. 1997. Neutrophils can adhere via alpha(4)beta(1)-integrin under flow conditions. Blood. 89:3837-3846

49. Baron, J.L., Madri, J.A., Ruddle, N.H., Hashim, G., and Janeway, C.A., Jr. 1993. Surface expression of alpha 4 integrin by CD4 T cells is required for their entry into brain parenchyma. J. Exp. Med. 177:57-68.

50. van Dinther-Janssen, A.C., et al. 1991. The VLA-4/VCAM-1 pathway is involved in lymphocyte adhesion to endothelium in rheumatoid synovium. J. Immunol. 147:4207-4210.

51. Laffon, A., et al. 1991. Upregulated expression and function of VLA-4 fibronectin receptors on human activated $\mathrm{T}$ cells in rheumatoid arthritis. J. Clin. Invest. 88:546-552.

52. Baron, J.L., Reich, E.P., Visintin, I., and Janeway, C.A., Jr. 1994. The pathogenesis of adoptive murine autoimmune diabetes requires an interaction between alpha 4 -integrins and vascular cell adhesion molecule-1. J. Clin. Invest. 93:1700-1708.

53. Catalina, M.D., Estess, P., and Siegelman, M.H. 1999. Selective requirements for leukocyte adhesion molecules in models of acute and chronic cutaneous inflammation: participation of E- and P- but not L-selectin.
Blood 93:580-589.

54. Ferguson, T.A., and Kupper, T.S. 1993. Antigen-independent processes in antigen-specific immunity. A role for alpha 4 integrin. J. Immunol. 150:1172-1182

55. Chisholm, P.L., Williams, C.A., Lobb, R.R. 1993. Monoclonal antibodies to the integrin alpha- 4 subunit inhibit the murine contact hypersensitivity response. Eur. J. Immunol. 23:682-688.

56. Silber, A., et al. 1994. Recruitment of lymphocytes during cutaneous delayed hypersensitivity in nonhuman primates is dependent on Eselectin and vascular cell adhesion molecule 1. J. Clin. Invest. 93:1554-1563.

57. Issekutz, T.B. 1993. Dual inhibition of VLA-4 and LFA-1 maximally inhibits cutaneous delayed-type hypersensitivity-induced inflammation. Am. J. Pathol. 143:1286-1293.

58. Bargatze, R.F., Jutila, M.A., and Butcher, E.C. 1995. Distinct roles of Lselectin and integrins a 4b7 and LFA-1 in lymphocyte homing to Peyer's Patch-HEV in situ: the multistep model confirmed and refined. Immunity. 3:99-108.

59. Luscinskas, F.W., Ding, H., and Lichtman, A.H. 1995. P-selectin and vascular cell adhesion molecule 1 mediate rolling and arrest, respectively, of CD4+ T lymphocytes on tumor necrosis factor alpha-activated vascular endothelium under flow. J. Exp. Med. 181:1179-1186.

60. Jung, U., and Ley, K. 1999. Mice lacking two or all three selectins demonstrate overlapping and distinct functions for each selectin. J. Immunol. 162:6755-6762.

61. Oppenheimer-Marks, N., Davis, L.S., Bogue, D.T., Ramberg, J., and Lipsky, P.E. 1991. Differential utilization of ICAM-1 and VCAM-1 during the adhesion and transendothelial migration of human $T$ lymphocytes. J. Immunol. 147:2913-2921.

62. Hwang, S.T., et al. 1996. GlyCAM-1, a physiologic ligand for L-selectin, activates beta 2 integrins on naive peripheral lymphocytes. J. Exp. Med. 184:1343-1348.

63. Warnock, R.A., Askari, S., Butcher, E.C., and von Andrian, U.H. 1998 Molecular mechanisms of lymphocyte homing to peripheral lymph nodes. J. Exp. Med. 187:205-216. 